\title{
Alterações auditivas e vestibulares associadas ao uso da mefloquina: uma revisão integrativa
}

\author{
Auditory and vestibular changes associated with the use of \\ mefloquine: an integrative review
}

\author{
Anna Maria de Lira Cabral' $\mathbb{B}^{\mathrm{D}}$, Mônyka Ferreira Borges Rocha ${ }^{1}$ (D), Danielle Samara Bandeira Duarte ${ }^{1}$ (D),

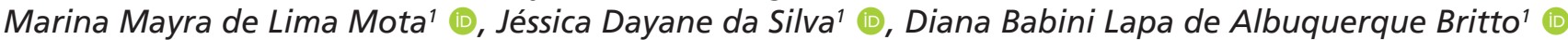

\section{RESUMO}

Objetivo: descrever, por meio de revisão da literatura, alterações auditivas e/ou vestibulares relacionadas ao uso em curto ou em longo prazo da mefloquina. Estratégia de pesquisa: trata-se de uma revisão integrativa, realizada nas seguintes bases de dados: PubMed, Web of Science, SciELO, LILACS, Scopus, ScienceDirect, Cochrane Library, Embase, OpenGrey, DissOnline e OAlster. Critérios de seleção: foram incluídos estudos com participantes a partir de 18 anos de idade, que fizeram uso de mefloquina e que foram submetidos à avaliação auditiva e/ou questionário referente à função auditiva e vestibular. Foram excluídas revisões de literatura, capítulos de livros e estudos que utilizaram a mefloquina combinada a outros medicamentos. Resultados: foram identificados 1.267 estudos nas bases de dados utilizadas, sendo selecionados 28 artigos para leitura completa. Destes, 12 foram incluídos na revisão, de acordo com os critérios de elegibilidade. Quatro artigos apontaram a presença de alterações vestibulares e auditivas, 2 indicaram apenas alterações auditivas e 6 apenas desordens vestibulares. No que se refere às manifestações auditivas, zumbido e perda auditiva foram os sintomas mais frequentes. Vertigem/tontura e desequilíbrio corresponderam às alterações vestibulares comumente apresentadas. Conclusão: manifestações auditivas e vestibulares foram referidas em curto e longo prazo, após o tratamento com a droga. A descontinuação de seu uso possibilitou a reversão das manifestações, porém, em alguns casos, foi observada a permanência das afecções. Considera-se importante a realização de acompanhamento audiológico e vestibular durante a ingestão da mefloquina, visto o seu perfil de toxicidade e possíveis manifestações colaterais de caráter auditivo e vestibular.

Palavras-chave: audição; perda auditiva; doenças vestibulares; vertigem; mefloquina

\begin{abstract}
Purpose: To describe through a literature review auditory and/or vestibular alterations associated with the short or long-term use of mefloquine. Research strategy: Integrative review performed on the following databases: Pubmed, Web of Science, Scielo, Lilacs, Scopus, Science Direct, Cochrane Library, Embase, Open Grey, DissOnline, OAlster. Selection Criteria: The articles selected included studies with participants that were 18 years old or over, who used mefloquine and who were submitted to an auditory evaluation and/or a questionnaire regarding auditory and vestibular function. Literature reviews, book chapters, and studies using mefloquine associated with other drugs were excluded. Results: 1,267 studies were identified in the databases used, 28 articles were selected for full reading, and out of these, twelve were included in the review according to the eligibility criteria. Four articles pointed out the presence of vestibular and auditory diseases, two indicated only auditory disorders, and six solely vestibular disorders. Regarding auditory manifestations, tinnitus and hearing loss (HL) were the most frequent symptoms. Vertigo/dizziness and imbalance matched to the vestibular changes were commonly observed. Conclusion: Auditory and vestibular manifestations were referred to in the short and long-term after treatment with the drug. The discontinuation of its use made it possible to reverse the manifestations; however, in some cases, the permanence of the disorders was reported. Audiological and vestibular follow-up during mefloquine use is considered important, given its toxicity profile and possible side manifestations of an auditory and vestibular nature.
\end{abstract}

Keywords: hearing; hearing loss; vestibular diseases; vertigo; mefloquine

\footnotetext{
Trabalho realizado no Departamento de Fonoaudiologia, Universidade Federal de Pernambuco - UFPE - Recife (PE), Brasil.

${ }^{1}$ Universidade Federal de Pernambuco - UFPE - Recife (PE), Brasil.

Conflito de interesses: Não.

Contribuição dos autores: AMLC, MFBR, DSBD, MMLM, JDS e DBLAB participaram da formulação da ideia central do estudo, definição de tema, objetivos, definição de população, intervenção e desfecho dos estudos a serem inseridos, critérios de inclusão, exclusão, traçado de chaves de busca e início da pesquisa; AMLC e DSBD realizaram a busca dos artigos nas bases científicas e literatura cinzenta; JDS atuou como terceiro avaliador em casos de discordâncias quanto à inclusão; MFBR e MMLM realizaram a síntese dos dados dos estudos incluídos; DBLAB fez a revisão desses dados; AMLC, MFBR, DSBD, MMLM, JDS e DBLAB realizaram a escrita do texto do artigo; DBLAB, MMLM e JDS fizeram a revisão do texto.

Financiamento: O presente trabalho foi realizado com apoio da Coordenação de Aperfeiçoamento de Pessoal de Nível Superior - Brasil (CAPES) - código de financiamento 001 .
}

Autor correspondente: Diana Babini Lapa de Albuquerque Britto. E-mail: diana.babini@gmail.com

Recebido: Agosto 14, 2020; Aceito: Novembro 12, 2020 


\section{INTRODUÇÃO}

Casos de doenças relacionadas ao sistema respiratório começaram a surgir com maior frequência nos hospitais de Wuhan (China), no fim de dezembro de 2019 e, em apenas um mês, 9.692 casos dessa disfunção foram confirmados. A partir de análises de amostras do líquido broncoalveolar de um dos pacientes, pôde-se confirmar que a infecção respiratória foi causada por um novo tipo de coronavírus. Diante disso, a doença passou a ser chamada de Covid-19, abreviação para Coronavirus Disease 19, ou SARS-CoV-2, abreviação para Severe Acute Respiratory Syndrome Coronavirus $2^{(1,2)}$.

Por se tratar de um novo vírus com alta capacidade de reprodução, a doença se alastrou por diversos países de forma rápida, tornando-se causadora de um estado de emergência global, declarado pela Organização Mundial de Saúde (OMS) ${ }^{(3,4)}$. O surto pandêmico e a rápida corrida por estratégias antivirais específicas no combate ao vírus, além de testes de medicamentos para o tratamento e prevenção da doença, têm sido o foco de ensaios e pesquisas clínicas. Desta forma, a administração de novos medicamentos em pacientes infectados pelo SARS-CoV-2 está sendo realizada em grande escala, a fim de controlar as complicações causadas pela Covid-19(5-7).

Um dos fármacos que tem sido descrito no uso de doenças respiratórias por SARS-CoV e como possível droga potencial contra o SARS-CoV-2 é o composto mefloquina (MQ) ${ }^{(8,9)}$. É conhecido como um medicamento antimalárico e parasitário, desenvolvido em 1970 e considerado como um análogo sintético do composto quinino, pertencente ao grupo aromático 4-quinolinemetanol. Ambas as substâncias são consideradas tradicionalmente ativas na profilaxia e tratamento da malária em humanos, causada pelo Plasmodium Falciparum, resistente ao composto de cloroquina ${ }^{(10)}$.

Em estudo realizado na China, pesquisadores relataram que o cloridrato de MQ provocou inibição completa dos efeitos citopáticos na cultura de células e sugeriu que o medicamento fosse considerado na investigação de estratégias terapêuticas adicionais no tratamento da infecção por Covid-19(11).

A Rússia foi o país pioneiro no teste de um medicamento à base de MQ no tratamento de infectados pelo novo coronavírus, apresentando, em março de 2020, um regime de tratamento à base da substância para o combate eficaz ao SARS-CoV-2 no organismo humano e com ação promissora na prevenção da doença $a^{(12)}$. Entretanto, o composto tem sido associado a uma gama de efeitos adversos de origem neurológica, em usuários profiláticos da substância, sendo comumente relatados a vertigem, dificuldades visuais e efeitos idiossincráticos, como distúrbios de equilíbrio, neuropatia periférica, parestesia, tremores e $\operatorname{ataxia}^{(13)}$.

Embora o uso da MQ tenha benefícios como droga antiviral, estudos a respeito de medicamentos antimaláricos utilizados contra infecções virais citam que a neurotoxicidade deve ser levada em consideração, devido à probabilidade de graves reações adversas ${ }^{(14,15)}$.

Considerando a toxicidade da MQ, presume-se que alterações na função auditiva e/ou disfunções vestibulares também possam ser causadas pelo uso desse medicamento antimalárico.

Tendo em vista a diversidade de medicamentos testados na atual pandemia de Covid-19 e a necessidade de conhecimento sobre os efeitos adversos, sejam auditivos e/ou vestibulares, relacionados ao uso desses fármacos, considera-se relevante esta revisão.

\section{OBJETIVO}

A presente revisão integrativa teve como objetivo descrever alterações auditivas funcionais e/ou vestibulares relacionadas ao uso em curto ou em longo prazo da MQ, em jovens, adultos e idosos.

\section{ESTRATÉGIA DE PESQUISA}

A revisão foi direcionada pela seguinte pergunta norteadora: "Qual o efeito da MQ utilizada em curto ou em longo prazo, nas vias auditivas funcionais e/ou vestibulares em humanos?"

A estratégia PICo foi utilizada e definida da seguinte forma: População (P): indivíduos que fizeram uso da MQ; Intervenção (I): avaliação funcional audiológica e/ou vestibular, bem como autorrelato de queixas audiológicas ou vestibulares; Contexto (Co): alterações funcionais auditivas e/ou vestibulares, bem como autorrelato de queixas auditivas e/ou vestibulares.

Este estudo foi realizado por meio das principais bases de dados disponíveis nos meses de maio e junho de 2020, especificamente: PubMed, Web of Science, Scientific Eletronic Library Online (SciELO), Literatura Latino-Americana e do Caribe em Ciências da Saúde (LILACS), Scopus, ScienceDirect, Cochrane Library e Embase, incluindo também as seguintes bases de dados para a Literatura Cinzenta: OpenGrey, DissOnline e OAlster.

Não foram aplicadas restrições de idioma e data de publicação. Com o objetivo de alcançar maior quantidade de artigos, foram utilizadas duas chaves de busca, associadas aos conectores booleanos AND e OR, sendo elas: mefloquine AND hearing OR hearing loss OR hearing diseases OR hearing disorders OR deafness; mefloquine AND vestibular diseases OR labyrinth diseases OR vertigo. Todos os descritores utilizados nas chaves de busca foram obtidos na base Medical Subject Headings (MeSH).

\section{CRITÉRIOS DE SELEÇÃO}

Dois revisores independentes selecionaram os artigos, inicialmente pela leitura do título e resumo e, finalmente, pela leitura do artigo por completo, de acordo com critérios preestabelecidos de inclusão e exclusão. Discrepâncias em relação à seleção e extração de dados foram discutidas entre os revisores no fim de cada etapa, objetivando chegar a um consenso e, na ausência de concordância, um terceiro avaliador foi consultado.

Foram incluídos estudos sem restrição quanto ao tipo de desenho, porém, que apresentassem: (1) descrição de pacientes humanos jovens, adultos e idosos, com idade a partir de 18 anos, que utilizaram MQ e que foram submetidos a qualquer tipo de avaliação auditiva e/ou questionário de autopercepção, relacionados à função auditiva e vestibular; (2) e/ou hipóteses ou evidências sobre a fisiopatologia do comprometimento auditivo associado ao uso do medicamento. Os critérios de exclusão adotados foram: revisões de literatura; capítulos de livros; estudos que usaram a MQ combinada a outro medicamento sem separações dos efeitos adversos para cada medicamento; estudos com indivíduos que já possuíam alterações da função auditiva e/ou vestibular. 


\section{ANÁLISE DE DADOS}

Os revisores, de maneira independente, extraíram os dados dos artigos selecionados em formato digital, sendo eles: o título do artigo, nomes dos autores, ano de publicação, país, tipo e objetivo do estudo, tamanho da amostra, faixa etária do grupo estudado, medicamentos utilizados, tempo de uso dos medicamentos, alterações audiológicas, alterações vestibulares, principais conclusões disponibilizadas pelos estudos e nível de evidência. Para classificar os artigos incluídos de acordo com o nível de evidência científica, foi utilizada a nova pirâmide de medicina baseada em evidências ${ }^{(16)}$.

Com o intuito de sintetizar a informação dos artigos, os dados extraídos dos estudos foram compilados de forma descritiva em uma tabela previamente elaborada, o que facilitou a identificação e reformulação das categorizações temáticas.

\section{RESULTADOS}

Foram identificados 1.267 estudos na busca inicial, sendo 52 selecionados após a leitura de títulos e resumos. Após remoção dos estudos duplicados, 28 artigos foram lidos na íntegra, conforme as etapas de seleção descritas (Figura 1). Por fim, 12 artigos foram selecionados após a exclusão dos que não atendiam aos critérios de elegibilidade preestabelecidos na metodologia. Os motivos de exclusão foram: artigos de revisão da literatura (5), alterações auditivas e/ou vestibulares associadas à doença de base e não ao medicamento utilizado (2), estudos realizados em animais (4), população investigada fora da faixa etária definida pela atual revisão (2), uso de outro tipo de medicamento associado (3).

Após a análise de todos os estudos incluídos na revisão integrativa, verificou-se ocorrência de 4 estudos experimentais, sendo 2 ensaios clínicos randomizados (nível de evidência 2) ${ }^{(17,18)}$ e 2 não randomizados (nível de evidência 3) ${ }^{(19,20)}, 4$ estudos observacionais (nível de evidência 4) ${ }^{(21-24)} \mathrm{e} 4$ estudos de casos (nível de evidência 5) ${ }^{(25-28)}$, de acordo com a classificação utilizada $^{(16)}$

Os estudos incluídos foram produzidos entre os anos de 1985 e 2017, nos continentes Europeu ${ }^{(19-22,25,27)}$, Americano ${ }^{(17,24,26,28)}$, Asiático $^{(23)}$ e Oceania ${ }^{(18)}$ (Quadro 1).

Os participantes dos estudos tinham idades entre 18 e 65 anos, sendo que, em 3 estudos, realizaram uso da MQ para tratamento da malária falciparum sintomática ${ }^{(17,25,27)} \mathrm{e}$, em 9 estudos, a administração do composto foi realizada em indivíduos saudáveis, como tratamento profilático da doençç ${ }^{(18-24,26,28)}$.

As doses orais ingeridas foram entre 100 e $6000 \mathrm{mg}$ de $\mathrm{MQ}$, variando em períodos de 1 dia a 6 meses de tratamento por via oral (Quadro 2). Verificou-se que 2 artigos apontaram a presença de alterações exclusivamente auditivas ${ }^{(25,26)}, 4$ estudos apresentaram alterações vestibulares e auditivas ${ }^{(18,19,23,27)} \mathrm{e}$ outros 6 , apenas alterações vestibulares ${ }^{(17,20-22,24,28)}$.

Em relação às manifestações auditivas comumente relatadas após o uso da MQ, foram descritos o zumbido ${ }^{(18,23,25-27)}$ e a perda

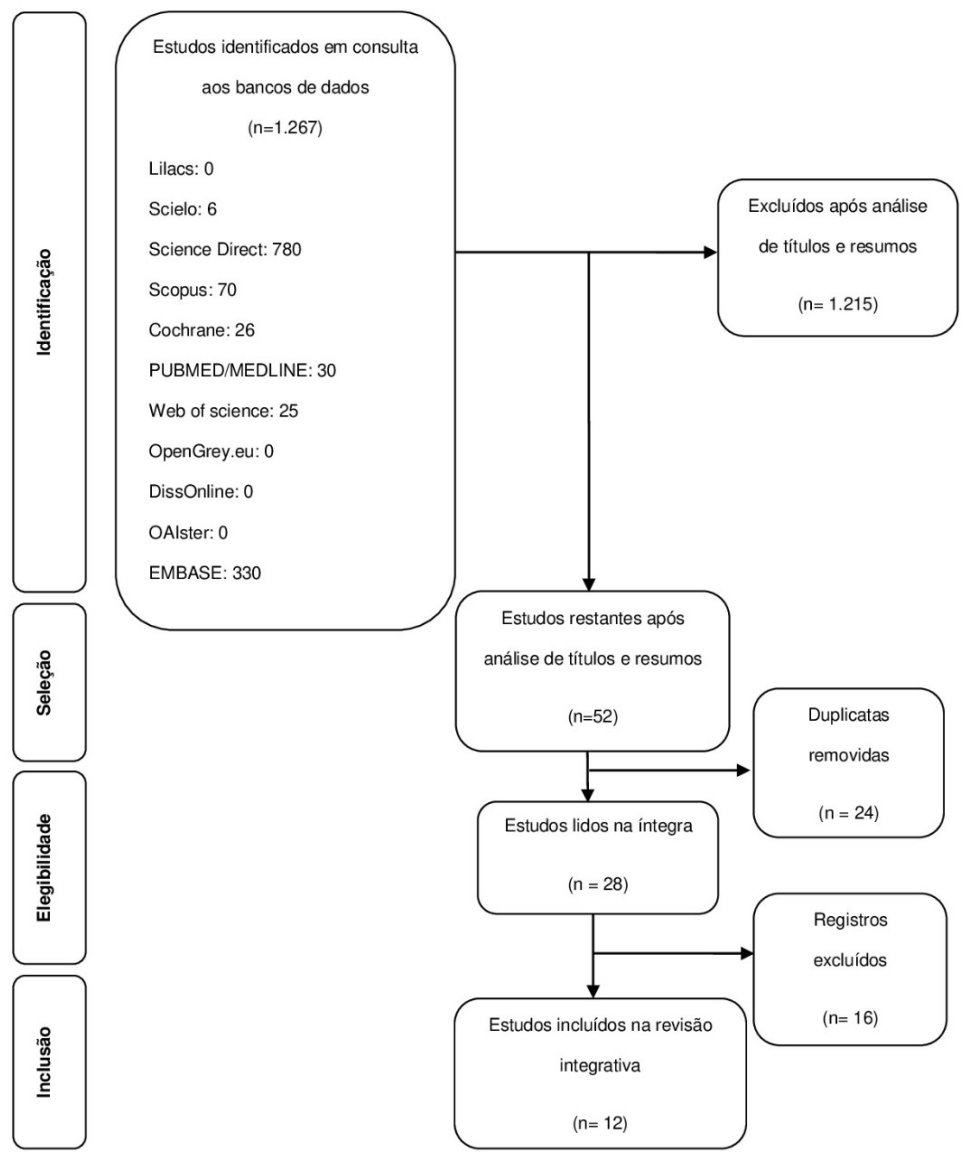

Figura 1. Diagrama de fluxo da seleção dos artigos

Legenda: $n=$ número de estudos 
Quadro 1. Descrição dos estudos incluídos na revisão integrativa da literatura

\begin{tabular}{|c|c|c|c|c|c|c|}
\hline Autor, ano & Título & Local & Objetivo & Tipo de estudo & Faixa etária & Amostra \\
\hline $\begin{array}{l}\text { De Souza et al. } \\
\text { (17) (1985) }\end{array}$ & $\begin{array}{l}\text { An open, randomized, } \\
\text { phase III clinical } \\
\text { trial of mefloquine } \\
\text { and of quinine } \\
\text { plus sulfadoxine- } \\
\text { pyrimethamine } \\
\text { in the treatment } \\
\text { of symptomatic } \\
\text { falciparum malaria in } \\
\text { Brazil }\end{array}$ & Brasil & $\begin{array}{l}\text { Comparar a segurança, } \\
\text { eficácia e efeitos colaterais } \\
\text { da } M Q \text { com o quinino mais } \\
\text { sulfadoxinapirimetamina, } \\
\text { em um período de } 3 \text { dias, } \\
\text { no tratamento da malária } \\
\text { falciparum. }\end{array}$ & $\begin{array}{l}\text { Ensaio clínico } \\
\text { randomizado }\end{array}$ & $\begin{array}{c}18 \text { a } 55 \\
\text { anos }\end{array}$ & 100 \\
\hline $\begin{array}{l}\text { Hessén- } \\
\text { Söderman } \\
(19) \\
(1995)\end{array}$ & $\begin{array}{l}\text { Mefloquine prophylaxis } \\
\text { and hearing, postural } \\
\text { control, and Vestibular } \\
\text { Functions }\end{array}$ & Suécia & $\begin{array}{l}\text { Estudar se a MQ afeta a } \\
\text { audição, a função vestibular } \\
\text { e o controle postural em } \\
\text { voluntários durante a profilaxia. }\end{array}$ & $\begin{array}{l}\text { Estudo de } \\
\text { intervenção não } \\
\text { randomizado }\end{array}$ & $\begin{array}{c}29 \text { a } 50 \\
\text { anos }\end{array}$ & 10 \\
\hline $\begin{array}{l}\text { Davis et al. }{ }^{(18)} \\
(1996)\end{array}$ & $\begin{array}{l}\text { Neurological, } \\
\text { cardiovascular and } \\
\text { metabolic effects of } \\
\text { mefloquine in healthy } \\
\text { volunteers: a double } \\
\text { blind, placebo- } \\
\text { controlled trial }\end{array}$ & Austrália & $\begin{array}{l}\text { Avaliar os efeitos colaterais da } \\
\text { administração da MQ em dose } \\
\text { profilática convencional em } \\
\text { voluntários saudáveis. }\end{array}$ & $\begin{array}{l}\text { Ensaio clínico } \\
\text { randomizado }\end{array}$ & $\begin{array}{l}\text { Média de } 24 \\
\text { anos }\end{array}$ & 106 \\
\hline $\begin{array}{l}\text { Van } \\
\text { Riemsdijk et al. }{ }^{(21)} \\
\text { (1997) }\end{array}$ & $\begin{array}{l}\text { Neuro-psychiatric } \\
\text { effects of antimalarials }\end{array}$ & Holanda & $\begin{array}{l}\text { Analisar os efeitos colaterais } \\
\text { neuropsiquiátricos das drogas } \\
\text { antimaláricas. }\end{array}$ & Coorte & $\begin{array}{c}38 \text { a } 42 \\
\text { anos }\end{array}$ & 394 \\
\hline $\begin{array}{l}\text { Fusetti et al. }{ }^{(25)} \\
(1999)\end{array}$ & $\begin{array}{l}\text { Mefloquine and } \\
\text { ototoxicity: A report of } \\
3 \text { cases }\end{array}$ & Itália & $\begin{array}{l}\text { Relatar os casos de pacientes } \\
\text { com uso da MQ para } \\
\text { tratamento da malária. }\end{array}$ & Estudo de caso & $\begin{array}{l}\text { Jovens e } \\
\text { adultos }\end{array}$ & 3 \\
\hline $\begin{array}{l}\text { Kollaritsch et al. } \\
\text { (22) }(2000)\end{array}$ & $\begin{array}{l}\text { Mefloquine } \\
\text { concentration profiles } \\
\text { during prophylactic } \\
\text { dose regimens }\end{array}$ & Áustria & $\begin{array}{l}\text { Relacionar as dosagens de } \\
\text { MQ e seus efeitos adversos } \\
\text { com o gênero. }\end{array}$ & Caso controle & $\begin{array}{l}\text { Média de } \\
29,2 \text { anos }\end{array}$ & 12 \\
\hline $\begin{array}{l}\text { Rendi- } \\
\text { Wagner et al. (20) } \\
\text { (2002) }\end{array}$ & $\begin{array}{l}\text { Unexpected frequency, } \\
\text { duration and spectrum } \\
\text { of adverse events after } \\
\text { therapeutic dose of } \\
\text { mefloquine in healthy } \\
\text { adults }\end{array}$ & Áustria & $\begin{array}{l}\text { Fornecer uma avaliação } \\
\text { confiável das reações } \\
\text { adversas associadas a } \\
\text { um regime terapêutico } \\
\text { antimalárico de MQ. }\end{array}$ & $\begin{array}{l}\text { Ensaio clínico } \\
\text { não randomizado }\end{array}$ & $\begin{array}{l}22 \text { a } 37 \\
\text { anos }\end{array}$ & 22 \\
\hline $\begin{array}{l}\text { Wise and } \\
\text { Toovey }^{(26)}(2007)\end{array}$ & $\begin{array}{l}\text { Reversible hearing } \\
\text { loss in temporal } \\
\text { association with } \\
\text { chemoprophylactic } \\
\text { mefloquine use }\end{array}$ & Canadá & $\begin{array}{l}\text { Discutir a possível etiologia da } \\
\text { relação entre PA e prescrição } \\
\text { da MQ. }\end{array}$ & Estudo de caso & 67 anos & 1 \\
\hline Nevin $^{(13)}(2012)$ & $\begin{array}{l}\text { Limbic encephalopathy } \\
\text { and central } \\
\text { vestibulopathy caused } \\
\text { by mefloquine: A case } \\
\text { report }\end{array}$ & Inglaterra & $\begin{array}{l}\text { Analisar o caso de um } \\
\text { paciente com vestibulopatia } \\
\text { central. }\end{array}$ & Estudo de caso & 24 anos & 1 \\
\hline $\begin{array}{l}\text { Livezey et al. } \\
\text { (2016) }\end{array}$ & $\begin{array}{l}\text { Prolonged } \\
\text { neuropsychiatric } \\
\text { symptoms in a military } \\
\text { service member } \\
\text { exposed to mefloquine }\end{array}$ & EUA & $\begin{array}{l}\text { Descrever a história clínica } \\
\text { de um homem de } 32 \text { anos } \\
\text { que desenvolveu sintomas } \\
\text { neuropsiquiátricos após uso } \\
\text { profilático da MQ. }\end{array}$ & Estudo de caso & 32 anos & 1 \\
\hline $\begin{array}{l}\text { Nevin and } \\
\text { Leoutsakos }^{(24)} \\
(2017)\end{array}$ & $\begin{array}{l}\text { Identification of a } \\
\text { Syndrome Class of } \\
\text { Neuropsychiatric } \\
\text { Adverse Reactions to } \\
\text { Mefloquine from Latent } \\
\text { Class Modeling of FDA } \\
\text { Adverse Event }\end{array}$ & EUA & $\begin{array}{l}\text { Identificar nova classe de } \\
\text { síndrome neuropsiquiátrica } \\
\text { associada ao uso da MQ. }\end{array}$ & Estudo seccional & $\begin{array}{c}18 \text { a } 65 \\
\text { anos }\end{array}$ & 933 \\
\hline
\end{tabular}

Legenda: $\mathrm{MQ}=$ Mefloquina; $\mathrm{PA}$ = Perda auditiva; $\mathrm{EUA}$ = Estados Unidos da América 
Quadro 2. Características relacionadas à condição de uso dos medicamentos e principais conclusões

\begin{tabular}{|c|c|c|c|c|c|c|}
\hline Autor/ano & Condição & Dose & $\begin{array}{l}\text { Tempo de } \\
\text { tratamento }\end{array}$ & $\begin{array}{c}\text { Alterações } \\
\text { Audiológicas }\end{array}$ & $\begin{array}{c}\text { Alterações de } \\
\text { equilíbrio }\end{array}$ & Principais conclusões \\
\hline $\begin{array}{l}\text { De Souza et al. } .^{(17)} \\
(1985)\end{array}$ & $\begin{array}{l}\text { Malária } \\
\text { Falciparum }\end{array}$ & $\begin{array}{l}100 \mathrm{mg} \text { de } \mathrm{MQ} \\
\text { por via oral em } \\
\text { dose única }\end{array}$ & 42 dias & - & $\begin{array}{l}16 \% \text { dos } \\
\text { participantes } \\
\text { apresentaram } \\
\text { tontura }\end{array}$ & $\begin{array}{l}\text { A tontura foi considerada um efeito } \\
\text { colateral leve e transitório, não } \\
\text { exigindo tratamento específico. Assim, } \\
\text { a MQ, foi considerada altamente } \\
\text { eficaz, segura e bem tolerada durante } \\
\text { o tratamento da malária falciparum } \\
\text { em homens adultos brasileiros. As } \\
\text { vantagens deste medicamento, que } \\
\text { pode ser administrado como uma } \\
\text { única dose via oral para o tratamento } \\
\text { do falciparum multirresistente malária, } \\
\text { são, portanto, óbvias. }\end{array}$ \\
\hline $\begin{array}{l}\text { Hessén- } \\
\text { Söderman(19) } \\
(1995)\end{array}$ & Profilaxia & $\begin{array}{l}250 \text { mg de } \mathrm{MQ} \\
\text { por via oral, } \\
\text { uma vez por } \\
\text { semana }\end{array}$ & 6 semanas & $\begin{array}{l}\text { Um paciente } \\
\text { apresentou } \\
\text { alteração, não } \\
\text { sendo especificada }\end{array}$ & $\begin{array}{l}\text { Um paciente } \\
\text { apresentou } \\
\text { tontura } \\
\text { inespecífica, leve } \\
\text { e constante }\end{array}$ & $\begin{array}{l}\text { Conclui-se que a MQ foi bem tolerada } \\
\text { e nenhum efeito geral da droga foi } \\
\text { visto no sistema postural. }\end{array}$ \\
\hline $\begin{array}{l}\text { Davis et al. } .^{(18)} \\
\text { (1996) }\end{array}$ & Profilaxia & $\begin{array}{l}1 \text { comprimido } \\
\text { por semana: } \\
250 \mathrm{mg} \text { de } \mathrm{MQ} \\
\text { ou } 250 \mathrm{mg} \text { de } \\
\text { placebo }\end{array}$ & 4 semanas & $\begin{array}{c}\text { Zumbido presente } \\
\text { em menos de } 10 \% \\
\text { dos indivíduos e } \\
\text { ausência de PA } \\
\text { significativa a } 6 \\
\mathrm{KHz}\end{array}$ & $\begin{array}{c}\text { Tontura em } \\
\text { menos de } 10 \% \\
\text { dos indivíduos }\end{array}$ & $\begin{array}{l}\text { Apesar da evidência de toxicidade } \\
\text { auditiva aguda do quinino em } \\
\text { indivíduos saudáveis, não foi } \\
\text { encontrada PA provocada pelo uso } \\
\text { da MQ. }\end{array}$ \\
\hline $\begin{array}{l}\text { Van } \\
\text { Riemsdijk et al. }{ }^{(21)} \\
(1997)\end{array}$ & Profilaxia & -- & 3 meses & - & $\begin{array}{l}\text { Vertigem, } \\
\text { tontura, visão } \\
\text { turva e ataxia }\end{array}$ & $\begin{array}{l}\text { Reações desfavoráveis ao uso da } \\
\text { MQ foram observadas. Grande parte } \\
\text { dos indivíduos usuários da droga } \\
\text { apresentou insônia, tontura, náusea, } \\
\text { diarreia, ansiedade, depressão, } \\
\text { palpitação e vertigem. Conclui-se que, } \\
\text { apesar de ser um medicamento com } \\
\text { diversos efeitos, a MQ ainda pode ser } \\
\text { considerada um medicamento útil para } \\
\text { o tratamento da malária. }\end{array}$ \\
\hline $\begin{array}{l}\text { Fusetti et al. }{ }^{(25)} \\
\text { (1999) }\end{array}$ & $\begin{array}{l}\text { Malária } \\
\text { Falciparum }\end{array}$ & - & - & $\begin{array}{l}\text { Zumbido e PA } \\
\text { sensorioneural de } \\
\text { alta frequência }\end{array}$ & - & $\begin{array}{l}\text { Um paciente apresentou remissão } \\
\text { parcial da PA após a suspensão da } \\
\text { MQ. Nenhum paciente relatou melhora } \\
\text { do zumbido. Sugerem avaliação } \\
\text { audiológica de rotina durante o uso } \\
\text { profilático de MQ para monitorar o } \\
\text { possível deficit auditivo. }\end{array}$ \\
\hline $\begin{array}{l}\text { Kollaritsch et al.(22) } \\
(2000)\end{array}$ & Profilaxia & $\begin{array}{c}6 \text { doses de } \\
250 \mathrm{mg} \text { de } \mathrm{MQ}\end{array}$ & 28 dias & - & Vertigem & $\begin{array}{l}\text { As reações adversas foram mais } \\
\text { frequentes em mulheres. Dor de } \\
\text { cabeça, insônia e vertigem foram } \\
\text { os efeitos colaterais mais comuns. } \\
\text { A menor tolerabilidade da } \mathrm{MQ} \\
\text { em mulheres pode ser devida às } \\
\text { concentrações mais altas de drogas } \\
\text { nesse grupo, indicando a necessidade } \\
\text { de um ajuste apropriado da dose de } \\
\mathrm{MQ} \text { em mulheres. }\end{array}$ \\
\hline $\begin{array}{l}\text { Rendi- } \\
\text { Wagner et al. }{ }^{(20)} \\
(2002)\end{array}$ & Profilaxia & $\begin{array}{l}1250 \text { mg de } \\
\text { MQ em } 5 \\
\text { comprimidos } \\
\text { de } 250 \mathrm{mg}, \\
\text { administrados } \\
\text { inicialmente } \\
\text { três } \\
\text { comprimidos } \\
\text { e seis horas } \\
\text { depois outros } \\
\text { dois. }\end{array}$ & 21 dias & - & $\begin{array}{l}\text { Vertigem } \\
\text { presente em } \\
96 \% \text { dos } \\
\text { indivíduos, } \\
\text { sendo do tipo } \\
\text { grave em } 73 \%\end{array}$ & $\begin{array}{l}\text { A vertigem foi descrita como tontura } \\
\text { associada a movimentos rápidos, } \\
\text { causando problemas na coordenação, } \\
\text { náuseas e vômitos graves, iniciando } \\
\text { dentro de } 24 \text { horas e atingindo o } \\
\text { pico no primeiro dia. Tais achados } \\
\text { representam a primeira investigação } \\
\text { com doses terapêuticas, permitindo } \\
\text { a identificação de reações adversas } \\
\text { associadas à } M Q \text {, independentemente } \\
\text { de quaisquer sintomas da malária. }\end{array}$ \\
\hline $\begin{array}{l}\text { Mizuno et al. }{ }^{.23)} \\
(2006)\end{array}$ & Profilaxia & - & 2 semanas & Zumbido & Tontura & $\begin{array}{l}\text { Aponta-se a importância de conhecer } \\
\text { o perfil de toxicidade e efeitos } \\
\text { adversos do uso prolongado de MQ. }\end{array}$ \\
\hline
\end{tabular}

Legenda: $\mathrm{MQ}=$ Mefloquina; $\mathrm{PA}=$ Perda auditiva 


\begin{tabular}{|c|c|c|c|c|c|c|}
\hline Autor/ano & Condição & Dose & $\begin{array}{l}\text { Tempo de } \\
\text { tratamento }\end{array}$ & $\begin{array}{c}\text { Alterações } \\
\text { Audiológicas }\end{array}$ & $\begin{array}{c}\text { Alterações de } \\
\text { equilíbrio }\end{array}$ & Principais conclusões \\
\hline $\begin{array}{l}\text { Wise and } \\
\text { Toovey } \\
{ }^{(26)}(2007)\end{array}$ & Profilaxia & $\begin{array}{l}3 \text { doses de } \\
250 \mathrm{mg} \text { de } \mathrm{MQ} \\
\text { (uma dose por } \\
\text { semana) }\end{array}$ & 21 dias & $\begin{array}{c}\text { Zumbido e PA } \\
\text { sensorioneural a } \\
90 \mathrm{~dB} \text { em } 1 \mathrm{kHz} \text { e } \\
70 \mathrm{~dB} \text { em } 4 \mathrm{kHz}\end{array}$ & - & $\begin{array}{l}\text { O caso pode fornecer uma lição sobre } \\
\text { contraindicações para uso de } M Q \text {, } \\
\text { sendo prudente evitar o uso da droga } \\
\text { em indivíduos com deficiência auditiva }\end{array}$ \\
\hline Nevin ${ }^{(13)}(2012)$ & $\begin{array}{l}\text { Malária } \\
\text { Falciparum }\end{array}$ & $\begin{array}{l}3 \text { doses de } \\
\mathrm{MQ}\end{array}$ & 15 dias & Zumbido & $\begin{array}{l}\text { Vertigem e } \\
\text { desequilíbrio }\end{array}$ & $\begin{array}{l}\text { A MQ é a causadora das alterações } \\
\text { de equilíbrio. }\end{array}$ \\
\hline $\begin{array}{l}\text { Livezey et al. }{ }^{(28)} \\
(2016)\end{array}$ & Profilaxia & $\begin{array}{c}250 \mathrm{mg} \text { por } \\
\text { semana }\end{array}$ & 6 meses & - & $\begin{array}{l}\text { Tontura, } \\
\text { desequilíbrio e } \\
\text { vertigem }\end{array}$ & $\begin{array}{l}\text { O estudo documenta o potencial } \\
\text { aparecimento de efeitos colaterais } \\
\text { neuropsiquiátricos induzidos por } \\
\mathrm{MQ} \text {, variando de uma vestibulopatia } \\
\text { central a alterações comportamentais } \\
\text { significativas, apresentando, também, } \\
\text { distúrbios do sono. }\end{array}$ \\
\hline $\begin{array}{l}\text { Nevin and } \\
\text { Leoutsakos }^{(24)} \\
(2017)\end{array}$ & Profilaxia & - & - & - & $\begin{array}{l}\text { Vertigem e } \\
\text { tontura }\end{array}$ & $\begin{array}{l}\text { O aparecimento de sintomas } \\
\text { neurológicos, como tontura, vertigem } \\
\text { e parestesias pode ajudar a melhorar } \\
\text { a descoberta de casos de estudos } \\
\text { com MQ sobre reações adversas } \\
\text { graves da droga. É importante que seu } \\
\text { uso seja descontinuado, caso haja } \\
\text { manifestações desses sintomas. }\end{array}$ \\
\hline
\end{tabular}

Legenda: $\mathrm{MQ}=$ Mefloquina; $\mathrm{PA}=$ Perda auditiva

auditiva $(\mathrm{PA})^{(18,19,25,26)}$. Quanto às afecções vestibulares, foram descritas a vertigem e/ou tontura ${ }^{(15,18-24,27,28)}$, sendo associadas, também, ao desequilíbrio ${ }^{(27,28)}$.

\section{DISCUSSÃO}

A MQ é um medicamento amplamente conhecido por sua atividade antimalárica, tanto para o tratamento da doença, quanto para uso profilático. Em todos os estudos incluídos nesta revisão, a droga foi administrada por via oral. Os indivíduos que participaram das pesquisas eram, em sua maioria, jovens e adultos $^{(17-25,27,28)}$, sendo encontrado apenas um estudo realizado em idosos ${ }^{(26)}$.

Foram apontados sinais e sintomas de alterações auditivas e vestibulares, como zumbido, PA, vertigem/tontura e desequilíbrio nos indivíduos que utilizaram a MQ de forma profilática e/ou como tratamento da malária. Os sintomas auditivos podem surgir de imediato ou em curto prazo, como no primeiro dia após a ingestão do medicamento, considerado como o pico de concentração da droga ${ }^{(19,20,26)}$, uma semana após ${ }^{(18)}$ ou até mesmo após fim do tratamento ${ }^{(17,25)}$.

Dentre os estudos incluídos, cinco deles realizaram exame de audiometria nos participantes ${ }^{(18,19,25-27)}$ e três realizaram avaliação otoneurológica $^{(19,27,28)}$. A avaliação do sistema vestibular foi constituída, especificamente, por exame vestibular de nistagmo espontâneo e após movimentação de cabeça ${ }^{(19)}$, teste de cadeira rotativa ${ }^{(27,28)}$, videonistagmografia, teste optocinético e de controle motor $^{(27)}$ e avaliação do reflexo vestibulococlear ${ }^{(28)}$.

Em relação aos efeitos colaterais ao uso da MQ estratificados por sexo, o feminino apresentou pontuação geral significativamente maior $^{(20,22)}$, comparado ao masculino, considerando a menor tolerabilidade da MQ em mulheres.

Quanto à remissão dos sintomas vestibulares, estes desapareceram após a descontinuação da medicação, com eliminação completa dos sintomas após seis semanas ${ }^{(19,20)}$. Em alguns casos, não foi observada remissão, havendo persistência de múltiplos episódios de vertigem e quedas ocasionadas por tontura, mesmo após quatro anos do fim do tratamento ${ }^{(28)}$, além da necessidade de encaminhamento para reabilitação vestibular, com suspeita de lesão nos núcleos oculomotor e vestibular ${ }^{(27)}$.

No tocante às alterações audiológicas, tanto há possibilidade de remissão dos sintomas ${ }^{(19,25,26)}$, como inalterações da condição auditiva, mesmo após interrupção do tratamento medicamentoso ${ }^{(25,27)}$.

Apesar dos efeitos adversos encontrados, existe relativa segurança do uso profilático da MQ em pessoas saudáveis ${ }^{(18)}$, considerando a droga tolerada em alguns indivíduos estudados, por não apresentarem significância nas manifestações auditivas e vestibulares encontradas ${ }^{(19)}$. No entanto, apesar de não terem sido observadas evidências de toxicidade auditiva associada à MQ em alguns estudos, foi apontado que possíveis alterações auditivas de baixo grau $(<5 \mathrm{~dB})$ não sejam identificadas de acordo com a etapa de teste utilizada ${ }^{(18)}$.

Para indivíduos em tratamento da malária, a MQ administrada em dose oral única de $1.000 \mathrm{mg}$ foi considerada altamente eficaz e segura ${ }^{(17)}$, considerando a baixa porcentagem dos efeitos adversos observados. É importante ressaltar que, apesar de tais conclusões, todos os estudos identificaram manifestações auditivas e/ou vestibulares nos participantes e que o tamanho da amostra (relativamente pequeno) foi considerado pelos próprios autores.

Em contrapartida, estudos apontaram os riscos relacionados a esse medicamento e mencionaram que reações adversas à MQ podem ocorrer, mesmo em pacientes sem contraindicações ao uso da droga, além de que manifestações como tontura e/ ou vertigem podem acontecer após a ingestão de um único comprimido de $250 \mathrm{mg}^{(27)}$. Também existe advertência para o fato de a MQ ser conhecida por sua neurotoxicidade, sendo a ototoxicidade um efeito adverso dessa substância, considerando importante que seja evitada, sempre que possível, em indivíduos que já possuem $\mathrm{PA}^{(26)}$.

O tratamento com MQ deve ser descontinuado quando do aparecimento de sintomas, como tontura e/ou vertigem, possibilitando a reversão de tais manifestações ${ }^{(24)}$. A avaliação audiológica de rotina, antes e após o uso profilático de medicamentos antimaláricos, também deve ser considerada, 
com o desígnio de observar e investigar mais de perto possíveis deficit auditivos ${ }^{(25)}$.

Apesar de alguns estudos não confirmarem a ototoxicidade causada pela MQ, autores mencionaram ${ }^{(20)}$ que, em pacientes com malária, considerada uma doença potencialmente fatal e que necessita ser tratada, as reações reversíveis aos medicamentos podem ser consideradas relativamente aceitáveis em ambientes hospitalares controlados, podendo ser este o motivo de algumas pesquisas considerarem uma boa tolerabilidade da droga em humanos.

Além disso, o repouso no leito de pacientes com malária, em ambientes hospitalares, pode mascarar reações a medicamentos, como a vertigem e/ou tontura. Desta forma, as taxas de efeitos colaterais potencialmente associados ao uso da MQ provavelmente podem ter sido subestimadas no passado ${ }^{(20)}$.

Devido à limitação de estudos quanto à eficácia e toxicidade da MQ, o apoio médico para a indicação da droga em indivíduos saudáveis, ou para tratamento da malária ${ }^{(20)}$ é de grande importância, sendo necessário ponderar os riscos dos efeitos colaterais associados ao uso ${ }^{(23)}$.

Considerando a variabilidade no aparecimento dos efeitos ototóxicos, do tempo de manifestação dessas alterações, das limitações quanto ao conhecimento da toxicidade da $\mathrm{MQ}$ mostrados nos estudos supracitados e do uso em teste na pandemia de Covid-19, é necessário que novas pesquisas sejam realizadas, a fim de ampliar o conhecimento do impacto do uso deste fármaco, tanto no sistema auditivo, quanto no sistema vestibular.

\section{CONCLUSÃO}

Os dados disponíveis nesta revisão permitiram o conhecimento de evidências sobre as alterações auditivas e vestibulares associadas ao uso profilático da mefloquina e no tratamento da malária em humanos, sendo relatados PA e zumbido, além de manifestações vestibulares como a tontura/vertigem e desequilíbrio. As afecções foram referidas em curto e longo prazo, após o tratamento com a droga. A descontinuação de seu uso possibilitou a reversão das manifestações, porém, em alguns casos, foi observada a permanência das alterações.

O conhecimento desses efeitos adversos de caráter auditivo e vestibular, em decorrência do uso da mefloquina, pode auxiliar na escolha da substância, considerando o seu perfil de toxicidade e possíveis manifestações colaterais. Com isso, é necessário o alerta aos possíveis riscos inerentes ao uso da droga antes do tratamento, bem como a realização de acompanhamento audiológico e vestibular durante a sua ingestão.

\section{ACKNOWLEDGEMENTS}

Coordenação de Aperfeiçoamento de Pessoal de Nível Superior - Brasil (CAPES).

\section{REFERÊNCIAS}

1. Wang D, Hu B, Hu C, Zhu F, Liu X, Zhang J, et al. Clinical characteristics of 138 hospitalized patients with 2019 novel coronavirus-infected pneumonia in Wuhan, China. JAMA. 2020;323(11):1061-9. http:// dx.doi.org/10.1001/jama.2020.1585. PMid:32031570.

2. Kannan S, Shaik Syed Ali P, Sheeza A, Hemalatha K. COVID-19 (Novel Coronavirus 2019) - recent trends. Eur Rev Med Pharmacol Sci. 2020;24(4):2006-11. http://dx.doi.org/10.26355/eurrev_202002_20378. PMid:32141569.

3. Liu Y, Gayle AA, Wilder-Smith A, Rocklöv J. The reproductive number of COVID-19 is higher compared to SARS coronavirus. J Travel Med. 2020;27(2):taaa021. http://dx.doi.org/10.1093/jtm/taaa021.

4. WHO: World Health Organization. Coronavirus disease 2019 (COVID-19) Situation Report - 51 [Internet]. 2020 [cited 2020 May 1]. Available from: https://www.who.int/docs/defaultsource/coronaviruse/situationreports/20200311-sitrep-51-covid-19.pdf?sfvrsn=1ba62e57_10

5. Zhai P, Ding Y, Wu X, Long J, Zhong Y, Li Y. The epidemiology, diagnosis and treatment of COVID-19. Int J Antimicrob Agents. 2020;105955(5):105955. http://dx.doi.org/10.1016/j.ijantimicag.2020.105955. PMid:32234468.

6. Ahn DG, Shin HJ, Kim MH, Lee S, Kim HS, Myoung J, et al. Current status of epidemiology, diagnosis, therapeutics, and vaccines for novel Coronavirus Disease 2019 (COVID-19). J Microbiol Biotechnol. 2020;30(3):313-24. http://dx.doi.org/10.4014/jmb.2003.03011. PMid:32238757.

7. Jin Y, Yang H, Ji W, Wu W, Chen S, Zhang W, et al. Virology, epidemiology, pathogenesis, and control of COVID19. Viruses. 2020;12(4):372. http://dx.doi.org/10.3390/v12040372. PMid:32230900.

8. Dyall J, Coleman CM, Hart BJ, Venkataraman T, Holbrook MR, Kindrachuk J, et al. Repurposing of clinically developed drugs for treatment of Middle East respiratory syndrome coronavirus infection. Antimicrob Agents Chemother. 2014;58(8):4885-93. http://dx.doi. org/10.1128/AAC.03036-14. PMid:24841273.

9. Serafin MB, Bottega A, Foletto VS, da Rosa TF, Hörner A, Hörner R. Drug repositioning is an alternative for the treatment of coronavirus COVID-19. Int J Antimicrob Agents. 2020;55(6):105969. http://dx.doi. org/10.1016/j.ijantimicag.2020.105969. PMid:32278811.

10. Nevin RL. Idiosyncratic quinoline central nervous system toxicity: historical insights into the chronic neurological sequelae of mefloquine. Int J Parasitol Drugs Drug Resist. 2014;4(2):118-25. http://dx.doi. org/10.1016/j.ijpddr.2014.03.002. PMid:25057461.

11. Fan HH, Wang LQ, Liu WL, An XP, Liu ZD, He XQ, et al. Repurposing of clinically approved drugs for treatment of coronavirus disease 2019 in a 2019-novel coronavirus-related coronavirus model. Chin Med J (Engl). 2020;133(9):1051-6. http://dx.doi.org/10.1097/ CM9.0000000000000797. PMid:32149769.

12. FMBA: Federal Biomedical Agency. Russia's [Internet]. Presented a drug for the treatment of coronavirus infection. 2020 [cited 2020 Jun 15]. Available from: http://fmbaros.ru/press-tsentr/novosti/ detail/?ELEMENT_ID=38052\&sphrase_id=15694

13. Nevin RL. Investigating channel blockers for the treatment of multiple sclerosis: considerations with mefloquine and carbenoxolone. J Neuroim. 2012;243(1-2):106-7. http://dx.doi.org/10.1016/j.jneuroim.2011.12.016.

14. D’Alessandro S, Scaccabarozzi D, Signorini L, Perego F, Ilboudo DP, Ferrante $\mathrm{P}$, et al. The use of antimalarial drugs against viral infection. Microorganisms. 2020;8(1):85-111. http://dx.doi.org/10.3390/ microorganisms8010085. PMid:31936284.

15. Ramos-Martín V, González-Martínez C, Mackenzie I, Schmutzhard J, Pace C, Lalloo DG, et al. Neuroauditory toxicity of artemisinin combination therapies: have safety concerns been addressed? Am J Trop Med Hyg. 2014;91(1):62-73. http://dx.doi.org/10.4269/ajtmh.13-0702. PMid:24865683. 
16. Murad MH, Asi N, Alsawas M, Alahdab F. New evidence pyramid. Evid Based Med. 2016;21(4):125-7. PMid:27339128.

17. de Souza JM, Sheth UK, de Oliveira RM, Roulet H, de Souza SD. An open, randomized, phase III clinical trial of mefloquine and of quinine plus sulfadoxine-pyrimethamine in the treatment of symptomatic falciparum malaria in Brazil. Bull World Health Organ. 1985;63(3):6039. PMid:3899397.

18. Davis TM, Dembo LG, Kaye-Eddie SA, Hewitt BJ, Hislop RG, Batty KT. Neurological, cardiovascular and metabolic effects of mefloquine in healthy volunteers: a double-blind, placebo-controlled trial. Br J Clin Pharmacol. 1996;42(4):415-21. http://dx.doi.org/10.1111/j.1365-2125.1996. tb00003.x. PMid:8904612.

19. Hessén-Söderman AC, Bergenius J, Palme IB, Bergqvist Y, Hellgren U. Mefloquine prophylaxis and hearing, postural control, and vestibular functions. J Trav Medic. 1995;2(2):66-9. http://dx.doi. org/10.1111/j.1708-8305.1995.tb00629.x.

20. Rendi-Wagner P, Noedl H, Wernsdorfer WH, Wiedermann G, Mikolasek A, Kollaritsch H. Unexpected frequency, duration and spectrum of adverse events after therapeutic dose of mefloquine in healthy adults. Acta Trop. 2002;81(2):167-73. http://dx.doi.org/10.1016/S0001706X(01)00210-8. PMid:11801224.

21. Van Riemsdijk MM, van der Klauw MM, van Heest JAC, Reedeker FR, Ligthelm RJ, Herings RMC, et al. Neuro-psychiatric effects of antimalarials. Eur J Clin Pharmacol. 1997;52(1):1-6. http://dx.doi. org/10.1007/s002280050240. PMid:9143859.

22. Kollaritsch H, Karbwang J, Wiedermann G, Mikolasek A, Na-Bangchang $\mathrm{K}$, Wernsdorfer W H. Mefloquine concentration profiles during prophylactic dose regimens. Wien Klin Wochenschr. 2000;112(10):441-7. PMID: 10890135.

23. Mizuno Y, Kudo K, Kano S. Chemoprophylaxis according to the guidelines on malaria prevention for Japanese overseas travelers. Southeast Asian J Trop Med Public Health. 2006;37(Suppl 3):11-4. PMid: 17547042.

24. Nevin RL, Leoutsakos J-M. Identification of a syndrome class of neuropsychiatric adverse reactions to mefloquine from latent class modeling of FDA adverse event reporting system data. Drugs R D. 2017;17(1):199-210. http://dx.doi.org/10.1007/s40268-016-0167-3. PMid:28063022.

25. Fusetti M, Eibenstein A, Corridore V, Hueck S, Chiti-Batelli S. Mefloquine and ototoxicity: a report of 3 cases. La Clinica Terapeutica. 1999 Sep-Oct;150(5):379-82.

26. Wise M, Toovey S. Reversible hearing loss in temporal association with chemoprophylactic mefloquine use. Travel Med Infect Dis. 2007;5(6):385-8. http://dx.doi.org/10.1016/j.tmaid.2007.08.006. PMid:17983978.

27. Nevin RL. Limbic encephalopathy and central vestibulopathy caused by mefloquine: a case report. Travel Medicine and Infectious Disease. 2012 Apr;10(3):144-51. http://dx.doi.org/10.1016/j.tmaid.2012.03.006.

28. Livezey J, Oliver T, Cantilena L. Prolonged neuropsychiatric symptoms in a military service member exposed to mefloquine. Drug Safety Case Reports. 2016 Jun;3(1):1-6. http://dx.doi.org/10.1007/s40800016-0030-z. 